\title{
Estratégias para a implementação de um projeto de pós-graduação em Educação e Saúde na Faculdade de Medicina da Universidade Federal do Rio Grande do Sul
}

PALAVRAS-CHAVE:

- Educação Médica Continuada;

-Educação Profissional emSaúde Pública;

- Reeducação Profissional;

-Educação Médica.

KEY-WORDS:

- Education, Medical, Continuing;

- Education, Public Health Professional;

- Retraining, professional

- Medical Education.

Recebido em: 07/02/2006

Reencaminhado em: 15/02/2007

Reencaminhado em:31/08/2007

Aprovado em: 11/10/2007
Strategies for implementation of a Graduate Program in Education and Health in the Medical School of the Federal University of Rio Grande do Sul

\author{
Waldomiro Carlos Manfroi ${ }^{1}$ \\ Carmen Lúcia Bezerra Machado ${ }^{1}$ \\ Malvina do Amaral Dorneles \\ Eliana Cláudia Ribeiro ${ }^{2}$ \\ Ronaldo Bordin ${ }^{1}$
}

\section{RESUMO}

Descrevem-se as estratégias empregadas para implementar uma linha de pesquisa em Educação e Saúde nos nove programas de pós-graduação já consolidados na Faculdade de Medicina da UFRGS. A linha está centrada na organização do trabalho pedagógico nos processos de ensinar e de aprender e capacitar docentes para atender às necessidades de formação multiprofissional. A curto prazo, objetiva atender às necessidades das Diretrizes Curriculares no curso de graduação em Medicina; a médio prazo, à titulação e qualificação do corpo docente nestes campos temáticos; a longo prazo, à implementação de um programa de pós-graduação em Educação e Saúde, em nível de mestrado e doutorado. A primeira turma ingressou em agosto de 2004 (seis alunos de mestrado e seis de doutorado), e seus estudos vêm sendo realizados, tanto na teoria como na prática, com base na análise de experiências em Educação e Saúde.

\section{ABSTRACT}

The authors describe the strategies used for establishing a research line in Education and Health in addition to the nine master's and doctorate programs already existing in the Medical School of the Federal University of Rio Grande do Sul. The research line is centered on organizing the pedagogical activity as refers to the teaching and learning processes as well as on qualifying teachers for meeting the demands of multi-professional education. The Project aims at meeting, in the short run, the need for Curricular Guidelines in the undergraduate medical course; in the middle run at qualifying the teaching body for these subjects; and in the long run, at implementing a Graduate Program in Health and Education on master and PhD levels. The first group of students was selected in August 2004 and their studies are being conducted, both theoretically and practically, based on the analysis of experiences on Education and Health. 


\section{INTRODUÇÃO}

A partir da perspectiva e das estratégias geradas pela metodologia da medicina baseada em evidência, surgiu a colaboração da Educação Médica Baseada na Melhor Evidência (Embe), em forma de rede internacional. Seu objetivo prioritário é recolher e promover o nível de maior evidência para colocar à disposição dos professores e das instituições, a fim de avaliar o rigor e a qualidade dos atuais e dos novos métodos de ensino e avaliação, visando à formação cada vez mais qualificada de médicos ${ }^{1}$.

A prática docente dos profissionais graduados em Medicina se caracteriza por ser desempenhada, tradicionalmente, em três momentos distintos, mas integrados - a assistência, o ensino e a pesquisa -, com notável diferença de enfoque e de hierarquia entre as três no processo que permeia estes saberes e fazeres.

$\mathrm{Na}$ assistência, os professores de Medicina têm várias oportunidades para se manter atualizados em seu fazer médico. Na pesquisa, buscam sempre novas maneiras de compreender e desenvolver a prática médica, promovendo um processo permanente de capacitação, até se tornarem pesquisadores independentes. Na docência, como demonstraram Batista ${ }^{2}$ e a Pesquisa do Cinaem ${ }^{3}$, entre tantas outras, a grande maioria dos professores das escolas médicas brasileiras não tem preparo pedagógico. Para completar este quadro de tríplice atuação, ao assumirem sua função docente, parecem abandonar todo o enfoque crítico sobre o que funciona e o que não funciona, orientando-se pela tradição e pela intuição ${ }^{4}$.

De outra parte, esses docentes tendem a salientar em demasia o dito: "se somos bons médicos, ensinamos bem". Nesse sentido, Ceccim e Feuerwerker ${ }^{5}$ ( p.140) afirmam que na educação "como setor específico das políticas públicas, não se observa a discussão da docência e do ensino-aprendizagem orientado para as profissões de saúde" e salientam que a área da formação é uma das mais carentes quanto à formulação de políticas para o Sistema Único de Saúde.

Os estudiosos da educação médica têm acompanhado os esforços da Associação Brasileira de Educação Médica (Abem), por meio da Rede de Apoio à Educação Médica (Raem), para organizar um cadastro da produção científica neste campo, disponibilizando instrumental teórico e metodológico que "auxilie a reflexão sobre o processo educativo", visando "apoiar a transformação da educação médica e estimular a produção científica sobre educação médica e áreas correlatas"

O empreendimento, certamente, enfrenta obstáculos semelhantes aos encontrados por Mauro ${ }^{7}$ em seu estudo exploratório sobre a pesquisa em educação médica, no qual apontou a ausência de sistematização da produção científica no campo da educação médica no País (denotando, de alguma forma, a baixa visibilidade desse campo de investigação) e salientou que a maioria das investigações nesse campo está fora da escola médica.

Esse autor, empregando uma série de descritores passíveis de mapear a produção do conhecimento na área de educação médica, encontrou 19 linhas vinculadas a 11 grupos de pesquisa que se ocupam do tema educação médica, dentro da escola médica. Esses grupos representam $1,4 \%$ da média dos grupos constituídos em escolas médicas; $0,6 \%$ da média dos grupos de Ciências da Saúde; 0,2\% da média dos grupos de Ciências da Vida; e 0,1\% da média dos grupos em nível nacional no período analisado 7 .

Uma das maneiras de modificar esta situação é investir tempo e esforço no desenvolvimento de projetos de investigação em Educação e Saúde para dispor de métodos educativos inovadores que possam ser avaliados com suficiente evidência científica ${ }^{3-8}$. Encontram-se em andamento diversas opções de mudanças, incluindo-se reformas curriculares, experiências novas de ensinar, umas testadas, outras em curso, em diversos centros nacionais e internacionais sobre educação em saúde. Essas experiências têm contribuído para o exercício de um novo olhar no processo ensino-aprendizagem, por incorporarem, nas instituições, metodologias para o ensino ativo, contribuindo para a implementação da mudança de paradigma na formação dos profissionais da saúde ${ }^{9-30}$.

Acresce-se a esses princípios a imperiosa necessidade que as escolas médicas têm de se adaptar às Diretrizes Curriculares do Ministério da Educação, que substituem o Currículo Mínimo por variáveis de competências e modificam substancialmente as metodologias e os cenários de ensino, privilegiando o trabalho em pequenos grupos, centrado no aluno, e empregando o sistema de avaliação formativa, para atender ao perfil do médico necessário:

[...] formação geral, capacitando-o a promover $e$ proteger a saúde da gestante, da criança, do adulto e do idoso; prestar assistência aos problemas de saúde de maior prevalência na população e encaminhar adequadamente os de nível mais complexo; tomar medidas necessárias à preservação da vida em situações de urgência médica; estabelecer uma relação profissional adequada com pacientes, famílias e comunidade, respeitando normas éticas; conhecer os princípios básicos de planejamento, organização e direção dos serviços de saúde; avaliar trabalhos científicos, elaborar e executar projetos de pesquisa e continuar sua educação médica de forma autônoma ${ }^{31}$. 
Este texto descreve as estratégias adotadas para implantar um projeto de pós-graduação em Educação e Saúde na Faculdade de Medicina da Universidade Federal do Rio Grande do Sul (UFRGS). O projeto foi implementado para suprir a carência de conhecimento nestas áreas do saber, em três situações caracterizadas como prioritárias para a melhoria da qualidade de ensino do curso médico: a curto prazo, atender às necessidades das Diretrizes Curriculares no curso de graduação em Medicina; a médio prazo, à titulação e qualificação do corpo docente nestes campos temáticos; a longo prazo, à implementação de um programa de pósgraduação em Educação e Saúde, em nível de mestrado e doutorado.

\section{A FACULDADE DE MEDICINA DA UFRGS - BREVE CARACTERIZAÇÃO}

A Faculdade de Medicina da UFRGS, a terceira escola médica fundada no País, apresenta corpo docente qualificado e forte estímulo à produção do conhecimento, integrando graduação, programas de residência médica e nove programas de pós-graduação. Possui um programa de Educação Continuada consolidado ( $20^{\circ}$ ano), diversidade nas áreas de ensino para seus cursos de graduação em Medicina e Nutrição, e cursos de especialização estruturados segundo demandas identificadas junto aos gestores do Sistema Único de Saúde (SUS). Seus cursos de mestrado e doutorado se encontram consolidados (nível 5 ou superior da Capes), seguindo uma lógica da pesquisa clínica, à exceção do programa de pósgraduação em Epidemiologia, que mantém linhas de pesquisa relevantes relacionadas à produção de conhecimentos na área da saúde, com prioridade para aqueles com maior potencial de interesse do SUS.

O interesse pelo preparo pedagógico dos professores da área da saúde na UFRGS remonta ao início da década de 1970, quando da realização dos Seminários de Ensino Médico, desenvolvidos na Faculdade de Medicina e, posteriormente, da implementação do Curso de Especialização para Professores Universitários da Área da Saúde, organizado pela Faculdade de Educação da UFRGS.

Com a implementação dos programas de pós-graduação da Faculdade de Medicina, em particular pela pós-graduação em Medicina: Cardiologia (1976), essa intenção se materializou na obrigatoriedade em cursar a disciplina Pedagogia Médica (quatro créditos). Em 1988, com a implementação do programa de pós-graduação em Clínica Médica, foram oferecidas mais duas disciplinas obrigatórias - Pedagogia Médica e Prática Didática -, demonstrando, mais uma vez, o interesse da Faculdade de Medicina na qualificação de seus docentes.
Com o incremento do número de programas de pósgraduação (PPGs) e a estruturação de uma comissão de pósgraduação, foi constituído, em 1997, um grupo de trabalho para promover estudos a fim de implementar uma nova disciplina ou ampliar as existentes que tratavam das questões pedagógicas de ensino médico. Com a participação de uma professora da Faculdade de Educação, criou-se uma disciplina obrigatória comum a todos os programas, denominada Prática Educativa em Medicina, com quatro créditos, semestral, com no máximo 25 alunos por turma, em atividade até hoje.

Entre setembro e novembro de 2001, a homologação das Diretrizes Curriculares do Ministério de Educação tornou mais evidente a necessidade de preparo pedagógico dos professores de Medicina para cumprir os novos desafios. Esta necessidade transpareceu nas metas apresentadas pela Faculdade de Medicina quando concorreu ao Edital do Programa de Incentivo às Mudanças Curriculares (Promed). Entre elas, destacamos o compromisso de estruturar uma linha de pesquisa em educação médica em todos os nove programas, como primeiro passo para conformar um programa de pósgraduação específico neste campo temático.

\section{A linha de pesquisa em Educação e Saúde - uma proposta de transição para um PPG específico}

Inserido no contexto do Promed, pelo qual a Faculdade de Medicina foi uma das 19 escolas contempladas, paralelamente às providências de reforma curricular e de adaptação a novos cenários de ensino, aprofundou-se a discussão sobre uma pós-graduação em educação médica. $\mathrm{O}$ primeiro passo envolveu a análise da possibilidade de implementação direta de um novo PPG, o que se revelou impossível em razão da inexistência de corpo docente qualificado nesta área, de modo compatível com os critérios mínimos preconizados pela Capes dentro da Faculdade de Medicina.

Uma segunda alternativa surgiu com a possibilidade de implantar uma área de concentração no PPG Educação, na Faculdade de Educação. Contudo, apesar de docentes de Medicina já terem cursado mestrado ou mesmo doutorado nesse programa, esta situação não prosperou por dificuldade de seleção dos candidatos aos temas componentes de linhas de pesquisa já estruturadas.

Destas duas possibilidades emergiu uma proposta que sintetizava as expectativas da Faculdade de Medicina em relação aos conhecimentos e experiência da Faculdade de Educação, entendida como um passo intermediário para um PPG específico: uma linha de pesquisa transversal a todos os PPGs da Faculdade de Medicina. Os alunos vinculados à mesma 
receberiam dois orientadores: um vinculado aos PPGs da Faculdade de Medicina, e outro proveniente do PPG Educação ou docente externo, convidado de outros PPGs nacionais (formalmente, um orientador e um co-orientador). O projeto, em seu formato inicial e com o título Pós-Graduação em Educação Médica, foi aprovado pela Comissão de PósGraduação em março de 2004 e pelo Conselho da Faculdade em junho desse mesmo ano ${ }^{32}$.

Para estruturar esta proposta, foi implementado um fórum de orientadores, agregando todos os potenciais docentes interessados em ofertar novas disciplinas na interface entre as áreas de saúde e educação. Em reuniões posteriores deste fórum, sua estrutura foi alterada para permitir o ingresso de outros profissionais da saúde, mudando-se o nome para Projeto de Pós-Graduação em Educação e Saúde. Reconheceuse, então, a dimensão educativa da qualificação e do exercício profissional na área da saúde e não apenas da educação médica em particular. Desta forma, a linha de pesquisa foi institucionalizada, alterando-se o nome original de "educação médica" para "educação e saúde".

\section{Foco de estudo da linha de pesquisa em Educação e Saúde}

O objeto central desta linha de pesquisa é o estudo de como vêm sendo efetivadas, na teoria e na prática, as experiências "de" e "em" Educação e Saúde, com vistas a formar educadores nessa área que superem o mito "bons médicos ensinam bem", criando condições para uma formação qualificada capaz de oferecer aos profissionais alternativas e inovações pedagógicas avaliadas cientificamente, em três eixos principais $^{31}$ :

Eixo 1: Organização do trabalho pedagógico. Centrado na instituição que promove a experiência, este eixo engloba o todo da proposta educativa, considerando a concepção de educação que sustenta o projeto, os princípios metodológicos, a base curricular, a presença dos pacientes na atividade assistencial inerente às aulas práticas, a proposta de avaliação, o trabalho dos diferentes setores, a organização dos educandos e as formas de gestão.

Eixo 2: Processos de ensinar e de aprender. Este eixo centrase nos ambientes educativos, com os sujeitos envolvidos (professores, educadores, trabalhadores, usuários e funcionários da saúde), no cotidiano das relações educativas, bem como nas diferentes linguagens, métodos e hierarquias.

Eixo 3: Relação com a comunidade científica, o Estado (em especial, MS e MEC) e os movimentos sociais nacionais e internacionais. Eixo centrado nas implicações da educação em saúde em suas relações externas à universidade, desenvolvidas com as organizações e os movimentos existentes na direção de aprofundar os estudos sobre as políticas públicas que permitam compreender e intervir na formação do educador.

\section{Da institucionalização do processo de transição}

Para atender aos objetivos propostos, sejam de consolidação da linha de pesquisa, sejam de introdução de novos conteúdos que institucionalizassem a proposta de construção de um novo PPG, foram implementadas as seguintes estratégias:

a) Definição do corpo docente, responsável pela estruturação da linha de pesquisa e de disciplinas integradoras entre as áreas de Educação e Saúde. Este momento se concretizou pela indicação dos orientadores pelos diversos PPGs da Medicina, pelo PPG da Faculdade de Educação e pela aceitação de professores de outras instituições de ensino.

Estes docentes conformaram um grupo de trabalho que definiu a necessidade de consultoria externa, para auxílio na execução da tarefa proposta, e de estruturação de um conjunto de novas disciplinas que, potencialmente, formariam um núcleo do futuro PPG Educação (Educação e Saúde I, Educação e Saúde II e Seminário de Pesquisa em Educação e Saúde I e II). Estas disciplinas, ao lado das disciplinas obrigatórias de cada PPG em que o aluno se inseriria (Bioética, Bioestatística, Epidemiologia Clínica), responderiam por cerca de $60 \%$ dos créditos obrigatórios. O restante dos créditos seria obtido em um núcleo de disciplinas da Faculdade de Educação, oferecidas semestralmente.

b) Definição de uma turma piloto, para análise de viabilidade estratégica da proposta: pela característica dos docentes envolvidos, emergiu a proposta de destinar seis vagas para mestrado e outras seis para doutorado, sendo pactuado que cada futuro aluno receberia um orientador da medicina e um co-orientador da educação, ou vice-versa, na condição presencial ou à distância.

Para esta primeira turma, definiu-se que as dissertações ou teses deveriam abordar, preferencialmente, temas relacionados ao processo de mudanças curriculares, à introdução de referências pedagógicas ativas e ao desenvolvimento dos projetos do Núcleo de Apoio Pedagógico e Pesquisa em Educação da Faculdade de Medicina e da Faculdade de Educação. Os recursos financeiros para a organização administrativa seriam provenientes, inicialmente, do Projeto Promed ${ }^{31}$.

Ainda que, inicialmente, a proposta se destinasse aos docentes da Faculdade de Medicina, com o avançar das discussões e com o apoio da Faculdade de Educação, definiu-se 
que o projeto deveria se voltar à qualificação de docentes de cursos da área da saúde da UFRGS, bem como incorporar profissionais que atuassem em cenários de ensino de nossos alunos da graduação. Naquele momento, ficou estabelecido também que profissionais da rede municipal de atenção à saúde, especialmente em unidades do Programa Saúde da Família, e do Grupo Hospitalar Conceição poderiam concorrer às vagas abertas para ingresso da primeira turma. A incorporação destes profissionais configuraria uma interface academia-serviço potencializadora da interdisciplinaridade e que também agregaria as demandas oriundas do Sistema Único de Saúde.

\section{Seleção e ingresso da turma}

Definido o número de orientadores e de orientandos, passouse a buscar uma nova forma de incorporá-los aos diversos PPGs da Faculdade de Medicina. Optou-se por matricular os alunos nos cursos com os quais apresentassem maior afinidade (fosse por especialidade clínica, fosse por departamento de origem, fosse por ser o PPG de seu orientador). Esta forma de recepção dos alunos reforçaria a necessidade de integração entre os diversos programas, sob a gerência da Comissão de Pós-Graduação.

A seleção respeitou todos os procedimentos empregados pelos diversos programas, incluindo uma prova escrita, que continha questões fechadas sobre conhecimentos em saúde e questões abertas relacionadas à Educação e Saúde, além de entrevista e análise do currículo.

As atividades tiveram início em 27 de agosto de 2004, e este projeto contou com a participação de 16 orientadores originários da Faculdade de Medicina, 6 da Faculdade de Educação e 6 orientadores de outras universidades do País e do exterior. Dos 12 alunos, 8 eram professores da Faculdade de Medicina da UFRGS, 1 da Faculdade de Odontologia, 1 da Farmácia, 1 do Grupo Hospitalar Conceição e 1 da Secretaria Estadual de Saúde. Todos estavam cursando as disciplinas para obter os créditos necessários e desenvolvendo seus projetos de pesquisa em Educação e Saúde.

Definiu-se como forma direta de avaliar os resultados desta turma piloto: a) o desempenho dos professores da Faculdade de Medicina e de outras unidades em suas dissertações ou teses; $b$ ) o impacto na atuação dos professores nos núcleos que tratam das questões relacionadas às mudanças curriculares, visando à implementação das Diretrizes Curriculares; c) o impacto das mudanças introduzidas em seus serviços pelos profissionais da saúde da rede de serviços.

\section{RESULTADOS PRELIMINARES}

Após 24 meses da inserção destes alunos na linha de pesquisa, os objetivos a curto prazo começaram a se consolidar.
Todos os alunos cursaram as disciplinas, no decurso de 18 meses, e obtiveram os créditos necessários. Quatro alunos (um de mestrado e três de doutorado) já obtiveram a aprovação de seus projetos de pesquisa pelo Grupo de Pesquisa e PósGraduação e pela Comissão de Ética do Hospital de Clínicas de Porto Alegre, bem como a qualificação junto às comissões dos respectivos programas de pós-graduação. Os projetos dos outros oito alunos estão em fase adiantada de elaboração. Todos os alunos participam das atividades que se desenvolvem em suas unidades relacionadas às mudanças curriculares e à realização de seminários e oficinas sobre metodologias ativas de ensino e sistema de avaliação.

Uma conseqüência indireta já ocorre: enquanto o projeto se consolida e vai sendo conhecido, surgem pedidos de informação para abertura de novas vagas. Há uma lista de 25 candidatos para seleção de ingresso em nova turma, que deverá ser oferecida assim que os atuais candidatos estiverem próximos de suas defesas.

Quanto à implementação de um Programa de Pós-Graduação em Educação e Saúde, embora seja difícil prever a concretização deste objetivo, pode-se assegurar que há boas perspectivas num futuro próximo, pela aceitação que vem obtendo. Temos consciência de que sua viabilização depende de múltiplos fatores, uns institucionais e governamentais, outros ligados ao impacto das publicações e da atuação dos atuais alunos.

Temos a convicção de que o sucesso das mudanças curriculares propostas e em andamento na área da saúde depende da formação pedagógica dos professores. E que esses objetivos serão alcançados somente se houver definição política e investimento na qualificação do corpo docente e na produção de conhecimentos em Educação e Saúde. Como a pós-graduação é, inquestionavelmente, a esfera que responde a esses condicionantes e agrega legitimidade e reconhecimento científico no Brasil, é no seu interior que devem ser consolidadas iniciativas brasileiras semelhantes a este projeto.

\section{REFERÊNCIAS}

1. Centeno AM, Martinez Carretero JM. Inovaciones, investigacion y evidencias em education médica: La colaboración EMBE (Educación Médica Basada en la Mejor Evidencia). Educ Méd mar 2003; 6(1):32-6

2. Batista NA. Conhecimento, experiência e formação: do médico ao professor de Medicina. Estudo sobre a disciplina Formação Didático-Pedagógica em Saúde nos Cursos de Pós-Graduação da Unifesp/EPM. São Paulo; 1997. LivreDocência [Tese] - Universidade Federal de São Paulo.

3. Comissão Interinstitucional Nacional de Avaliação das Escolas Médicas. Avaliação das escolas médicas brasileiras; 1997 (Relatório do Modelo Pedagógico). 
4. Van der Vleuten COM. The need for evidence in education. Med Teach 2000; 22:246-50.

5. Ceccim RB, Feuerwerker LCM. Mudança na graduação das profissões de saúde sob o eixo da integralidade. Cad Saude Publica 2004; 20(5):1400-10.

6. Associação Brasileira de Educação Médica. Projeto Rede de Apoio à Educação Médica. [online]. Disponível em http: / / www.abem-educmed.org.br/ raem / participe.html.

7. Mauro, Fortunato. A produção científica dos docentes da Faculdade de Medicina da UFRJ e sua relação com o fazer pedagógico. Rio de Janeiro; 2005. Mestrado [Dissertação] - Universidade Federal do Rio de Janeiro.

8. Harden RM, Lilley PM. Best evidence medical education: the simple truth. Med Teach 2000: 22:117-9.

9. Peterson S. Time for evidence based medical education. BMJ 1999; 318:1223-24.

10. Dolmans DHJM, Schmidt HG. The advenles of problembased curricula. Postgraduate Medicine J 1996; 72:535-8.

11. Grave WS, Schmidt HG, Boshuizen HPA. Effecta of problem-based discussion on stuying a subsequent text: a randomized trial among first year medical students. Instructional Science 2001; 29:33-44.

12. Hemker $\mathrm{CH}$. Critical perceptions on problem-based learning. Adv Health Sci Educ Theory Pract 1998; 3(1): 71-6.

13. Marcodes E, Gonçalves EL. Educação Médica. São Paulo: Sarvier; 1998.

14. Ribeiro EC. Ensino/aprendizagem na escola médica. In: Marcodes E, Gonçalves EL. Educação Médica. São Paulo: Sarvier; 1998. p. 40-49.

15. URL:/ / www.bemecolaboration.org. [capturado dez. 2001].

16. Norman G. Reflections on BEME. Med Teach 2000; 22:141-4.

17. Newble DI, Jaeger K. The effect of assessment and examination on the learning of medical students. Med Educ 1983:17:165-71.

18. Feuerwerker L. Além do discurso de mudança na Educação Médica. São Paulo: Hucitec; 2002.

19. Lampert JB. Tendências de mudança na formação médica. São Paulo: Hucitec; Rio de Janeiro: Abem; 2002.

20. Almeida M. Educação de profissionais de saúde na América Latina. São Paulo: Hucitec; 1999.

21. Lane JK. Processo de ensino e de aprendizagem em Medicina. São Paulo: Fundo Editorial Bik; 2000.
22. Dias Sobrinho J, Ristoff DI, Moretti MT. Revista da Rede de Avaliação Institucional da EducaçãoSuperior (Campinas). 2001.

23. Dolmans DHJM. The effectiveness of PBL: the debate continues. Some concerns about the BEME-movement. Medical education 2003; 37:1129-30.

24. Manfroi WC, Machado CLB, Petersen AM, Spina MJP. Necessidades de referências pedagógicas para o ensino de cardiologia, no Curso de Graduação. Arq Bras Cardiol 2002; 78:271-5.

25. Lambert BJ. Na transição paradigmática da Educação Médica: o que o paradigma da integralidade atende que o paradigma flexneriano deixou de lado. Boletim Abem 2003 jul.-out. ; 31(4/ 5):18-20.

26. Komatsu RS. Aprendizagem baseada em problemas: sensibilizando o olhar para o idoso. São Paulo: Associação Brasileira de Educação Médica eSociedade Brasileira de Geriatria e Gerontologia do Estado de São Paulo; 2003.

27. Venturelli J. Education médica: nuevos enfoques, metas y métodos. Washington: Organizacion Panamericana de la Salud; 2003.

28. Maia JÁ. O ensino de cardiologia na graduação médica. Desafios Atuais. Arq Bras Cardiol 2004; 82(3):302-306.

29. Manfroi WC, Machado CLB, Ribeiro EC, Bordin R. Estratégias para um projeto de pós-graduação em Educação e Saúde. Olho Mágico 2005; 12(2):65-87.

30. Machado CLB, Manfroi WC. Prática educativa em Medicina. Porto Alegre: DaCasa; 2005.

31. Programa de Incentivo a Mudanças Curriculares em Cursos de Medicina. Brasília: Ministério da Saúde/ Ministério da Educação; 2002.

32. Universidade Federal do Rio Grande do Sul. Faculdade de Medicina. Relatório da Direção, período 25 abr. 2001 24 abr. 2005.

O projeto contou com o suporte do Programa de Apoio a Mudanças Curriculares do Ensino Médico (Promed) Ministérios da Saúde e da Educação e Opas.

\section{CONFLITOS DE INTERESSE}

Declarou não haver

\section{ENDEREÇO PARA CORRESPONDÊNCIA}

Waldomiro Carlos Manfroi

Rua Ramiro Barcelos, 2400 - 4o andar - Bairro Bom Fim

90035-903 - Porto Alegre - RS

E-mail: wmanfroi@hcpa.ufrgs.br 